\title{
Network Architecture for a High Bandwidth WDMA/CDMA Local Area Network
}

\author{
F. Ayadi, P. Fortier, E. Hamelin, B. Ruchet, L. A. Rusch, M. Têtu \\ (http://www.gel.ulaval.ca/ copgel) \\ Department of Electrical and Computer Engineering \\ Laval University, Québec, Canada G1K 7P4
}

\begin{abstract}
AвSTRACt - We propose an optical WDMA/CDMA hybrid high bandwidth local area network using coherent homodyne detection. Local injection locking to centralized highly stable sources is used for frequency selection and coherent detection. Splitting losses in a fully connect optical local area network are significant for large systems ( 1000 subscribers) necessitating the use of optical amplifiers. We examine several network configurations to optimize capacity while avoiding amplifier saturation and keeping the number of amplifiers required to a minimum.
\end{abstract}

\section{A WDMA/CDMA Local Area Network}

Local area networks currently provide at most $100 \mathrm{Mb} / \mathrm{s}$ of aggregate data transmission, while future video-intensive applications are expected to drive requirements for bit rates to $100 \mathrm{Mb} / \mathrm{s}$ per user. Existing network protocols and electronic transmission paths cannot be adapted to support this anticipated demand. Optical transmission paths provide the necessary bandwidth, however efficient protocols that can exploit this bandwidth are still the subject of research. We propose a flexible WDMA (wavelength division multiple access) -- CDMA (code division multiple access) hybrid system. The number of CDMA users at one wavelength is limited by the speed of electronic components that realize the spectral spreading $(\sim 10 \mathrm{~Gb} / \mathrm{s})$. For spreading codes of length 127 this corresponds to on the order of 10 active users. To increase system capacity multiple wavelengths are used, with a cluster of CDMA users operating at each wavelength. Requirements for dense packing of wavelengths are relieved by the ability of CDMA to increase system capacity at each wavelength. Several characteristics of this network set it apart from those proposed previously, including

- use of bipolar codes via phase modulation

- very narrow linewidth reference lasers

- injection locking for frequency selection \& purity

- homodyne coherent detection

We use bipolar codes for CDMA to take advantage of their enhanced auto-correlation and cross- correlation properties compared to those for $0 / 1$ codes used with on-off keying. This requires that the phase be modulated and in this paper we consider only external phase modulation. As described in [1], the phase can also be modulated by control of injection locking current, at the expense of a moderate penalty in performance.

This system uses a central bank of very narrow linewidth reference wavelengths for WDMA. These reference wavelengths are distributed to each user via a fiber bus independent of the fiber network for modulated data. Users would employ these reference signals to injection lock their distributed feedback (DFB) lasers for either transmission or reception. Activating one of a bank of DFB lasers with nominal center frequency on the desired wavelength provides wavelength selection. Injection locking ensures the DFB laser adopts the frequency purity and absolute frequency reference of the reference source, hence allowing for homodyne coherent detection.

\subsection{Network logical configuration}

Figure 1 illustrates how data is transferred from one user to another. The intended recipient has code number two on wavelength $i$. The first user activates the DFB laser with nominal center frequency $\lambda_{i}$, locking it to the signal from the reference bus. The output of the DFB laser is modulated externally by the data and spreading code two, and is then sent to all users via the data network. The second user decodes his message by also injection locking to $\lambda_{i}$ and phase modulating by code two. Only the proper combination of wavelength and code will despread the data. This network uses $W$ different wavelengths and a set of $D$ different codes, each one being assigned to a different user, for a total of $W D$ users in the network.

The logical network delivers the reference signals to each user via one fiber system and all modulated data signals via an independent fiber system. This network can be implemented in any number of physi- 


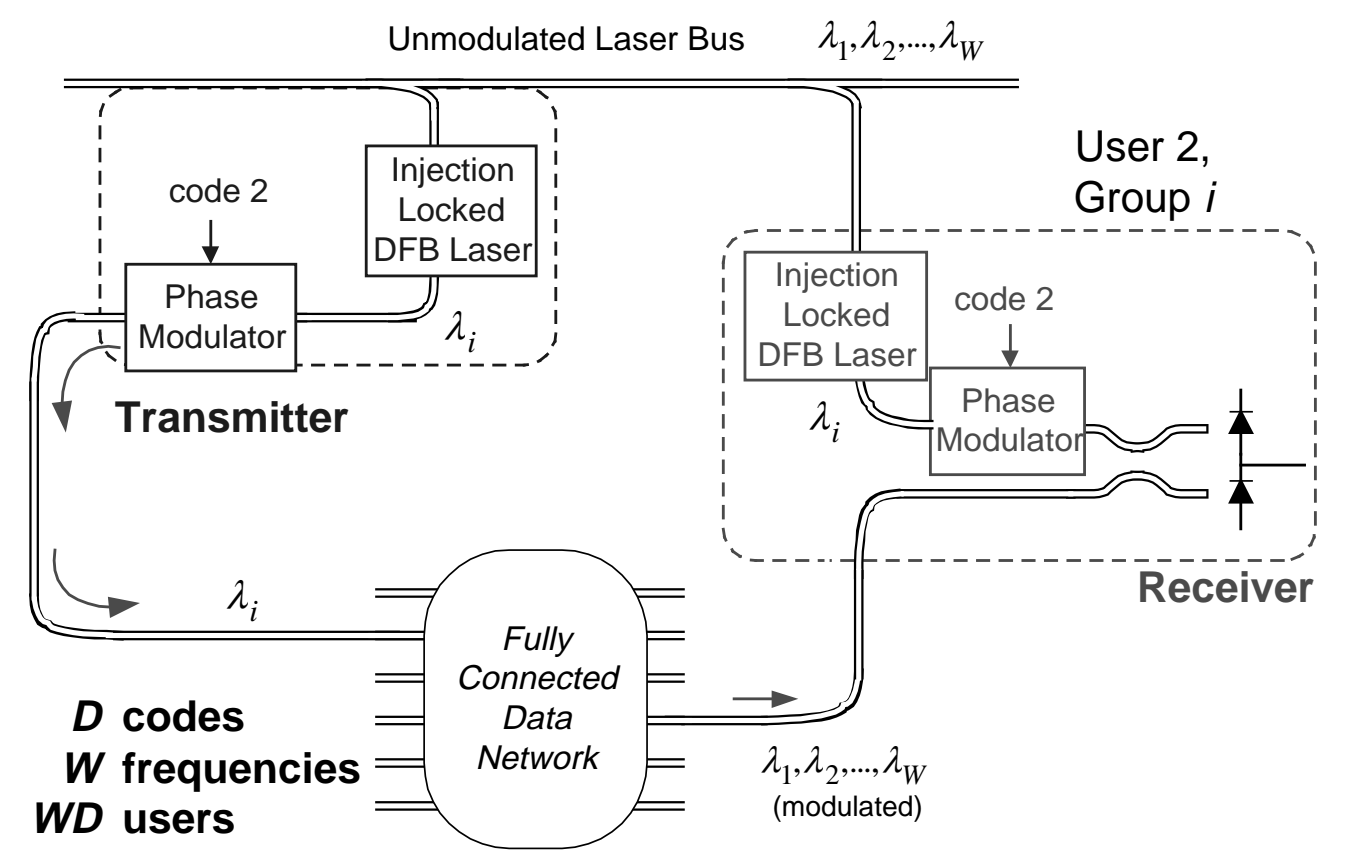

Figure 1 WDMA/CDMA Local Area Network - arbitrary user communicates with user employing code 2 and wavelength $\lambda_{i}$

cal configurations. We will choose a physical configuration maximizing the number of users while maintaining 1) a robust power budget, 2) low variation in the input power of the fiber amplifiers, and 3) equal power losses for all users, i.e., all users will have roughly equal received powers to avoid the socalled near-far problem. To determine the maximum number of stations that can be supported, some definitions and assumptions are in order. Let $M$ be the power margin (in $\mathrm{dB}$ ), defined as $M=10 \log \left(P_{T} / P_{R}\right)$ where $P_{T}$ is the available optical transmitter power and $P_{R}$ is the minimum receiver power that provides a tolerable bit-error rate (BER). Typically, $M$ is on the order of $40 \mathrm{~dB}$, representing the allowable losses in the network.

\subsection{Physical configuration options}

The configurations considered here are a bus, tree, star and compound topologies. The bus configuration has data from each station injected into the bus via directional couplers, and broadcasts to receivers. The useful power delivered to each receiver is reduced by $1 / \mathrm{N}^{2}$ for an $\mathrm{N}$-station network. A linear arrangement of $N$ taps has end-to-end loss (dB) [3]:

$$
L=-10 N \log (1-\alpha)-10 N \log \beta
$$

where $\alpha$ is the coupler power splitting ratio and $\beta$ is the coupler excess loss. For typical values $\alpha=0.1$ and $\beta=0.8$, a bus configuration is limited to a maximum of 8 users for $M=40 \mathrm{~dB}$. This configuration suffers from the near-far problem, that is, the receiver signal power for each user will not be equal.

The second basic configuration we consider is the minimum-depth binary-tree topology that allows delivery of uniform signal strength for all users. The excess loss for an $N$-station tree increases only logarithmically with $N$ with total

$$
L=2\left(10 \log N-10 \log \beta \frac{\log N}{\log 2}\right)
$$

For the same power margin $M=40 \mathrm{~dB}$, a maximum of 32 users is obtained. Thus the tree structure improves the situation (from 8 to 32 ), although the maximum number of supported stations is still too low to be of interest.

The third basic configuration is the star topology, where an $N \times N$ star coupler interconnects $N$ transmitters and receivers with an excess loss of

$$
L=10 \log N-10 \log \beta \frac{\log N}{\log 2}
$$

As is the case with the tree configuration, the first term of the above equation usually dominates, increasing logarithmically with $N$, while that of an $N$-station bus increases linearly with $N$. The difference in loss can be quite significant, especially for large values of $N$. The maximum number of users accommodated in such a configuration can reach up to 1024 users [3].

In addition to the basic configurations described, we can combine architectures to increase flexibility 
while maintaining network capacity. For instance, we can interconnect several trees with a passive modular star [4]. Each tree represents a group of different users sharing the same physical location such as a floor in a high-rise building, with the trees joined by a star coupler. For example, if we take the same power margin of $40 \mathrm{~dB}$, it is possible to accommodate 128 users using a tree-star-tree configuration by using a $16 \times 16$ star coupler and connecting each port to a tree with 8 branches. There are $\log _{2} N$ stages, each with approximately $4 \mathrm{~dB}$ of attenuation, for a total attenuation of $40 \mathrm{~dB}$ in the network.

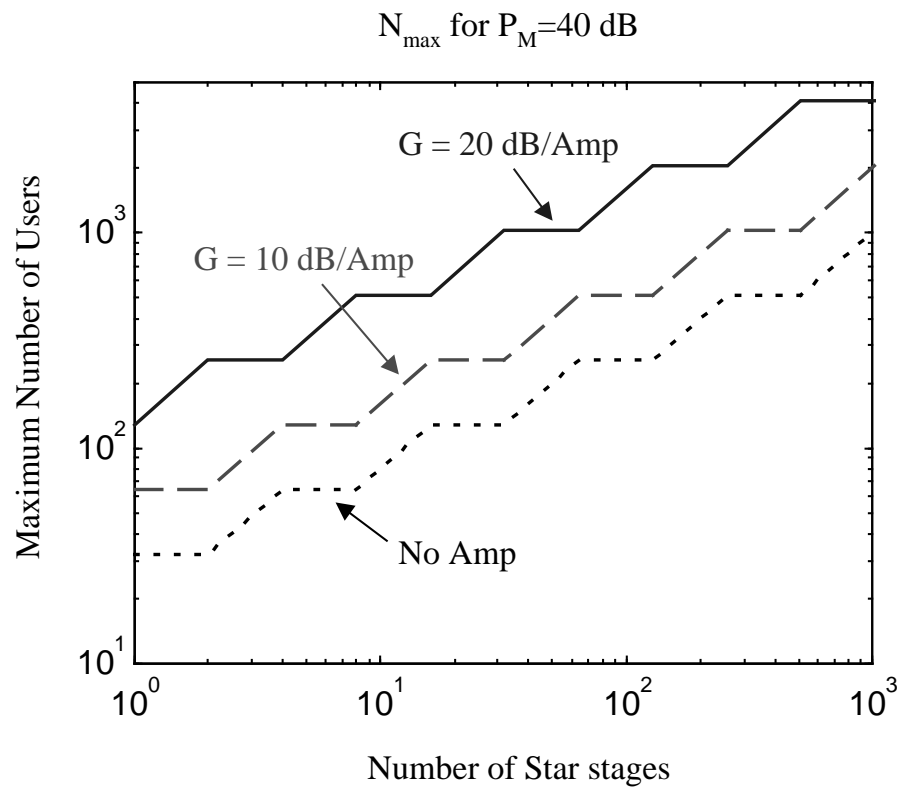

Figure 2 System capacity for various amplifier gains

The capacity of the compound configuration is greater than that of the tree configuration, but is less than the maximum obtained for the star coupler, which is the price of a more flexible configuration. This flexibility is important for placement of the optical amplifiers. Any practical system will require amplifiers, and these must be placed so as to ensure proper amplifier performance. The power at the input must not be too great in order to avoid saturation, while the total number of amplifiers should be controlled to hold down total system cost. Of course, amplifier placement should also be consistent with our goal of roughly equal received powers of all active users. Figure 2 shows how amplifiers can increase capacity using the tree-star-tree with allowable losses of $40 \mathrm{~dB}$.

In the proposed system the amplifiers are located as indicated in Figure 3. The tree structure ensures that each output of the star coupler will contain the sum signal of all active users, each with roughly

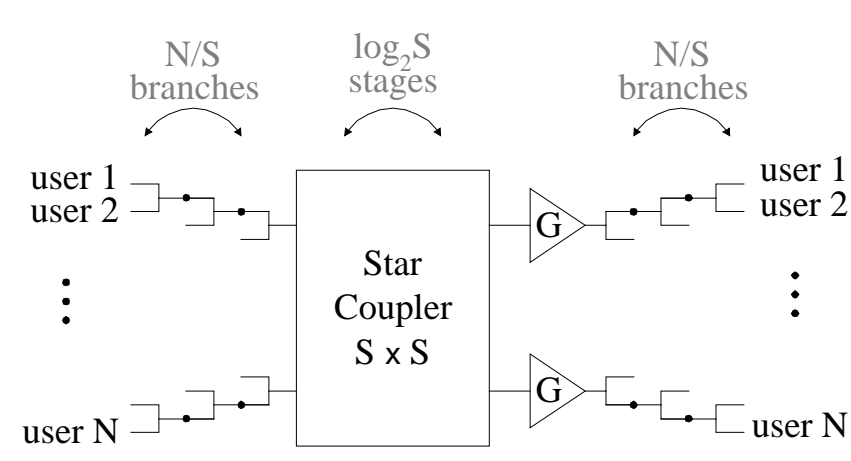

(transmitters)

(receivers)

Figure 3 Tree-Star-Tree configuration

identical powers. As not all users will be transmitting at any given time, it is possible that the power in the branches of the transmitting side of the tree will be highly unequal. However, the amplifiers are placed at the star coupler outputs so that each amplifier will see the sum of all active users, minimizing input power variability. Clearly the number of amplifiers required is determined by the size of the star coupler. The star coupler must be large enough so that power delivered at each output does not drive the amplifier into saturation, yet small to minimize the number of amplifiers required.

\subsection{Power budget}

In the following, we evaluate the maximum number of users, $N$, in the network as a function of the number of levels in a tree-star-tree network, where $S$ is the number of inputs/outputs of the star coupler, and $k=\log _{2} S$. Examining Figure 1 and Figure 3, we can summarize the system's powers and losses for one carrier wavelength using coherent homodyne detection. We call $P_{\text {laser }}$ the power output of the injection-locked DFB laser, with a typical value of $3 \mathrm{dBm}$. The phase modulator introduces attenuation $A t t_{\text {mod }}$ of about $4 \mathrm{~dB}$. We call $L_{\text {preamp }}$ the losses due to the tree (transmission side) and star coupler. From equations (2) and (3) this is given by

$$
\begin{aligned}
L_{\text {preamp }}= & 10 \log \frac{N}{S}-10 \log \beta \frac{\log N / S}{\log 2} \\
& +10 \log S-10 \log \beta \frac{\log S}{\log 2} \\
= & 10 \frac{\log N}{\log 2}(\log 2-\log \beta) \\
= & n \cdot 10(\log 2-\log \beta)=n \cdot 4 \mathrm{~dB}
\end{aligned}
$$

The optical amplifier has gain $G$. The tree on the reception side of the network introduces losses 


$$
\begin{aligned}
L_{\text {postamp }} & =10 \log \frac{N}{S}-10 \log \beta \frac{\log N / S}{\log 2} \\
& =10 \frac{\log N-\log S}{\log 2}(\log 2-\log \beta) \\
& =(n-k) \cdot 4 \mathrm{~dB}
\end{aligned}
$$

Finally we lump all fiber and polarization losses into the single term $L_{F}$. The polarization loss is on the order of $1 \mathrm{~dB}$, while the fiber losses over $10 \mathrm{~km}$ is about $2.5 \mathrm{~dB}$.

Having examined the system losses we now focus on the average power, $P_{S}$, required at the dual balanced receiver in order to maintain a bit-error rate (BER) of $10^{-9}$. Let $T$ be the transmission rate, $K$ be the maximum number of simultaneous users, $C$ be the length of the spreading code, and $R_{\lambda}$ be the receiver sensitivity at wavelength $\lambda$. The SNR of the dual balanced receiver is then given by [1]:

$$
S N R=\left[\frac{K-1}{3 C}+\frac{e T}{4 R_{\lambda} P_{S}}\right]^{-1}
$$

For homodyne demodulation, the probability of error is $1 / 2$ erfc $\sqrt{S N R / 2}$. For a $100 \mathrm{Mbit} / \mathrm{s}$ transmission rate, 10 simultaneous users, length 127 code and a receiver sensitivity of $R_{\lambda}=1.125 \mathrm{~A} / \mathrm{W}$ with $\lambda_{i}=1.55 \mu \mathrm{m}$, we need an average signal power of $P_{S}=-61 \mathrm{dBm}$ at the input of the dual balanced receiver to ensure a BER of $10^{-9}$.

We have hypothesized 10 active users per channel over 10 separate wavelengths. Using a 10\% utilization rate for the network, this means a total subscriber base of approximately $N=1024$ users, or $n=10$. We can now calculate the number of amplifiers and the amplifier gain required for various compound configurations, and ensure that the amplifiers will not be forced into saturation. Note from equation (4) that the configuration loss on the transmission side of the network is $40 \mathrm{~dB}$, independent of the tree-star-tree trade-off. This means that the amplifier input power will vary from $-44 \mathrm{dBm}$ for one active user to $-24 \mathrm{dBm}$ for the maximum of 100 active users (on all 10 wavelengths). We are therefore assured that the amplifiers will operate in the linear portion of the gain curve. Table 1 presents the number of amplifiers and the total gain required for various configurations.

\section{Conclusion}

We have proposed a network architecture for a hybrid WDMA/ CDMA system accommodating a total of 1024 users with a high flexibility in the choice of its physical configuration. This network configuration allows delivery of uniform signal strength for all users, a desirable feature in a CDMA system. We evaluated the power budget for different physical configurations of a system of 1024 users. We considered only systems where the optical amplifiers are at the output of the star coupler to maximize the stability of the input power. This system has a probability of error of $10^{-9}$ for as many as 100 users transmitting simultaneously at $100 \mathrm{Mb} / \mathrm{s}$ (10 different users per wavelength).

\section{References}

1. F. Ayadi and L. A. Rusch, "Coherent Optical CDMA with Limited Phase Excursion", IEEE Communications Letters, vol. 1, pp. 28-30, January, 1997.

2. D. Zaccarin and M. Kavehrad, "Performance Evaluation of Optical CDMA Systems Using NonCoherent Detection and Bipolar Codes," Journal of Lightwave Technology, Vol. 12, No. 1, Jan. 1994.

3. P. E. Green, Jr., Fiber Optic Networks, Prentice Hall, 1993.

4. J. F. Hayes and F. Ayadi, "Local Optical Distribution", INFOCOM'92 Conference Proceedings, pp. 68-74, May 1992.

Table 1 Amplifier requirements

\begin{tabular}{c|c|c|c}
\hline $\begin{array}{c}\text { Number } \\
\text { of star } \\
\text { stages }\end{array}$ & $\begin{array}{c}\text { Configuration } \\
\text { Tree-Star-Tree } \\
\mathbf{2}^{\mathbf{1 0 - k}}-\mathbf{2}^{\mathbf{k}}-\mathbf{2}^{\mathbf{1 0 - k}}\end{array}$ & $\begin{array}{c}\text { Gain for } \\
\mathbf{6 . 5} \mathbf{~ d B} \\
\text { margin }\end{array}$ & $\begin{array}{c}\text { Number } \\
\text { of Amp. } \\
\text { needed }\end{array}$ \\
\hline 0 & $1024-1-1024$ & $30 \mathrm{~dB}$ & 1 \\
\hline 1 & $512-2-512$ & $26 \mathrm{~dB}$ & 2 \\
\hline 2 & $256-4-256$ & $22 \mathrm{~dB}$ & 4 \\
\hline 3 & $128-8-128$ & $18 \mathrm{~dB}$ & 8 \\
\hline 4 & $64-16-64$ & $14 \mathrm{~dB}$ & 16 \\
\hline 5 & $32-32-32$ & $10 \mathrm{~dB}$ & 32 \\
\hline 6 & $16-64-16$ & $6 \mathrm{~dB}$ & 64 \\
\hline 7 & $8-128-8$ & none & none \\
\hline 8 & $4-256-4$ & none & none \\
\hline 9 & $2-512-2$ & none & none \\
\hline 10 & $1-1024-1$ & none & none \\
\hline \multicolumn{4}{|r}{}
\end{tabular}

